\title{
Transatlantica
}

Revue d'études américaines. American Studies Journal

Line Breaks in America: the Odds and Ends of Poetry

\section{Gilles Havard, L'Amérique fantôme : Les aventuriers francophones du Nouveau Monde}

Jean-Marc Serme

\section{OpenEdition}

1 Journals

Édition électronique

URL : https://journals.openedition.org/transatlantica/16663

DOI : 10.4000/transatlantica. 16663

ISSN : 1765-2766

Éditeur

Association française d'Etudes Américaines (AFEA)

Référence électronique

Jean-Marc Serme, « Gilles Havard, L'Amérique fantôme : Les aventuriers francophones du Nouveau Monde », Transatlantica [En ligne], 1 | 2021, mis en ligne le 01 juin 2021, consulté le 01 février 2023 URL : http://journals.openedition.org/transatlantica/16663; DOI : https://doi.org/10.4000/ transatlantica.16663

Ce document a été généré automatiquement le 1 février 2023.

Creative Commons - Attribution - Pas d'Utilisation Commerciale - Pas de Modification 4.0 International - CC BY-NC-ND 4.0

https://creativecommons.org/licenses/by-nc-nd/4.0/ 


\title{
Gilles Havard, L'Amérique fantôme : Les aventuriers francophones $d u$ Nouveau Monde
}

\author{
Jean-Marc Serme
}

\section{RÉFÉRENCE}

L'Amérique fantôme: Les aventuriers francophones du Nouveau Monde, Gilles Havard, Paris : Flammarion, 2019, 656 pages, ISBN : $9782082105163,26,00 €$.

\section{Introduction}

1 L'œuvre que construit ouvrage après ouvrage Gilles Havard se poursuit dans ce nouvel épisode de son exploration au long cours des relations franco-indiennes en Amérique $\mathrm{du}$ Nord, de l'arrivée des colons français jusqu'au milieu du XIX⿸e siècle. Depuis Empire et Métissage (2004) et plus récemment, dans son livre de 2016, Histoire des coureurs de bois. Amérique du Nord 1600-1840, dont on peut dire avec Allan Greer qu'il est « une étude majeure sur un personnage collectif au cœur de l'histoire de la traite des fourrures : le fameux coureur de bois » (Greer \$2), Gilles Havard poursuit sa déclinaison des concepts et thématiques qui ont trait au monde de la «mobilité pelletière » et du « phénomène social » qu'est la chasse aux animaux à fourrure (494). Cette nouvelle somme de plus de 500 pages de texte a pour argument central l'effacement, voire la "disgrâce " des coureurs de bois français dont l'aventure a été « négligée et même étouffée aux ÉtatsUnis » (14) en faveur de héros anglophones garants de la Destinée Manifeste tels que Kit Carson. Havard revisite ici des thématiques fondatrices qui l'obsèdent et qui revenaient encore dans son précédent opus comme la mobilité spatiale des biens et des personnes, les valeurs indiennes de générosité et de réciprocité, la masculinité des coureurs, les «loyautés multiples» envers les empires rivaux, les positions sociales de ces «figures 
fantômes » en quête ou déjà détentrices d'une certaine liberté face aux conventions et aux lois des puissants. Havard réitère son regret de voir l'Amérique française "effacée de l'histoire et des mémoires » (12). On peut cependant rappeler qu'un historien étatsunien comme Jay Gitlin, d'ailleurs cité dans la bibliographie (602), a publié en 2010 une étude sur la présence française aux États-Unis. Le même auteur fera paraître en août 2021 un recueil d'essais intitulé French St. Louis: Landscape, Contexts, and Legacy aux Presses de l'Université du Nebraska, dans la collection «France Overseas: Studies in Empire and Decolonization Series »'1. Peut-être faut-il y voir un frémissement d'intérêt dans le monde anglophone pour la présence française en Amérique dans les siècles passés?

2 Mais, ce qui est réellement fascinant dans le projet élaboré par Gilles Havard, c'est son exploration de «la forme d'indétermination de l'Amérique des coureurs de bois» (14) qui l'extirpe de "toute vision téléologique » de la conquête et dresse au contraire le portrait d'un continent ouvert aux avenirs possibles, et surtout, une Amérique amérindienne certes non épargnée par des catastrophes précoces, mais bien structurée par des visions du monde qui tentent d'intégrer les nouveaux-venus dans des schémas de compréhension autochtones, et ce jusqu'à très tard dans le XIX siècle. Ainsi, la patiente élaboration de cette "couche de francité", cette "strate francophone" greffée à «l'immense socle constitué par des centaines de langues autochtones » que l'auteur s'emploie à restituer, contribue à envisager « immédiatement » l'Amérique du Nord, comme il le plaide à la fin de son ouvrage, "dans sa dimension continentale ", c'est-à-dire ne pas retenir les schèmes étatsuniens « d'une historiographie fondée sur un mouvement d'est en ouest des colons » (502) qui compartimenterait a posteriori une géographie et une histoire sociale globales dont les échanges à très grandes distances organisaient depuis des siècles les rapports humains. Néanmoins, Havard n'est pas dupe du processus engagé par les puissances occidentales dans les Amériques et il ne tombe pas dans le piège de l'oublier ou de l'écarter pour renforcer sa thèse, comme il le craignait un peu dans sa recension du Middle Ground de Richard White en 2010 : «Pour invisible qu'il puisse parfois paraître (les Indiens sont loin d'être inféodés et demeurent des acteurs politiques autonomes), ce pouvoir [colonial] n'en est pas moins rampant ».

\section{La langue française}

La langue française est pour Havard l'idiome dont l'extension est jugée "presque continentale" durant plus d'un siècle, entre 1730 et 1850 (500). De Montréal aux Rocheuses, la langue française a joué un rôle important dans le développement des réseaux de traite des fourrures et il est bien le sujet de la réhabilitation que propose ici l'auteur. Si les Amérindien-ne-s n'ont jamais vraiment cherché à apprendre à parler français ou anglais, les échanges entre traiteurs étaient principalement francophones et construisaient une confrérie soudée et solidaire. Plus généralement, Havard montre comment la langue, les langues devraient-on dire, étaient capitales dans les rapports de sociabilité entre coureurs et groupes amérindiens. L'interprétariat fut un métier de longue haleine pour des générations de ces hommes et la maîtrise des idiomes amérindiens pouvait sauver la vie d'un non-autochtone, lui donner accès à la sociabilité tribale, voire à la parenté lorsqu'il s'unissait avec une amérindienne. Ces alliances donnèrent naissance à une classe de coureurs métis bilingues (ou polyglottes). « Métis » est aujourd'hui une des "classifications politiques canadiennes contemporaines" 
(Havard 9). Pendant des siècles donc, parler plusieurs langues, dont le français, apporta réputation et influence à des hommes souvent en marge de leur communauté d'origine et dans les sociétés amérindiennes dans lesquelles ils circulaient.

\section{Cheminements, itinéraires et carrefours (récits biographiques)}

4 La mobilité est le schème central dans ce livre. Les « exemples de cheminement » (16) qui y sont étudiés occupent des trajectoires est-ouest et retour, mais aussi des axes sudnord et retour. De la région laurentienne $d u x_{\text {XII }}{ }^{e}$ siècle à la baie des Puants (aujourd'hui la région de Green Bay, sur la rive occidentale du lac Michigan), de St Louis dans la seconde partie du XviII ${ }^{\mathrm{e}}$ siècle puis la vallée du Missouri jusqu'au Rocheuses, courant $\mathrm{XIX}^{\mathrm{e}}$ siècle, nous suivons des itinéraires fluviaux et équestres dans des mondes culturels différents, changeants, alliés ou ennemis.

5 L'approche biographique dans ce genre de recherche permet de suivre les déplacements au long cours, d'en mesurer la spécificité et l'épaisseur humaine, évitant ainsi de ramener les protagonistes "uniquement aux marqueurs collectifs des souscultures dans lesquelles ils se meuvent ", écrit Havard (492). Ce procédé de narration biographique a été utilisé par Theodore Catton en 2017 dans son récit croisé de trois acteurs anglophones (pour deux des trois au moins) du commerce de fourrures en déclin au début du xix ${ }^{e}$ siècle. "Le commerçant " John McLoughlin, «l'explorateur " Stephen H. Long et «le chasseur » John Tanner étaient alors réunis dans une histoire complexe dont le nœud est un comptoir commercial sur la frontière nord de l'état actuel du Minnesota, Rainy Lake House. L'auteur y déployait un récit haletant à partir des textes rédigés ou dictés par les trois protagonistes. Comme l'écrivait en 2002 l'historienne Naomi Wulf, la biographie permet « l'histoire d'une époque vue au travers de cet individu (ou groupe d'individus), qui n'est plus présenté comme un héros, mais comme une sorte de réceptacle, au carrefour d'événements, de courants ou de mouvements que son récit de vie rend parfois plus tangibles" (Wulf \$1). Dans ce contexte, Catton, comme Havard, propose à travers ses personnages de capter «la subjectivité de [leur] perception » et de relever « le défi de construire la vérité à partir de multiples points de vue» (5).

\section{Classes sociales et mobilité}

6 Ce qui intéresse également Havard est de voir comment les « frontières supposées entre les groupes sociaux» peuvent être discutées, voire traversées par ces personnages « subalternes» (241) aux prises avec les codes et les hiérarchies sociales de leur époque. Il rappelle la distinction entre les termes "coureur de bois» et "voyageur»: le premier date de 1672 et concerne «ceux qui sortent des seigneureries sans congé (permission) » et le second est un terme qui permet de « désigner plus noblement les individus " après 1680 (202). La course est un moyen de se créer des "espaces de liberté » ou au moins d'évoluer en "zones grises » (492) dans une société européenne très hiérarchisée, grâce à leur "expertise " $(490,495)$ utilisée comme levier social. Nombre de coureurs travaillent pour des compagnies pelletières, ou bien des commanditaires, des marchands installés dans des villes stratégiques qui organisent les 
saisons de traite. Montréal, Michillimakinak et St Louis sont trois des points névralgiques autour desquels gravitent les traiteurs étudiés par Havard. Beaucoup de ces hommes parviennent d'ailleurs, à l'instar de Jean-Baptiste Truteau, à intégrer au moins « la couche inférieure de la notabilité » (492) de ces villes en expansion.

\section{Masculinité}

7 La course de bois est un monde d'hommes dans un système à la fois compétitif de parts de marché, mais aussi de coopération dans un monde où l'entraide, l'échange de services, le soutien moral ou matériel étaient une question de vie ou de mort. Les degrés de masculinité se trouvent définis par les qualités physiques, linguistiques ou logistiques qui font la différence entre le succès et l'échec. Les valeurs masculines européennes rencontrent les visions amérindiennes, spécifiques selon les groupes, de ce qu'être un homme veut dire.

\section{Sociabilité et religion}

8 L'approche anthropologique, ethnohistorique pour être précis², de Gilles Havard, est à sa plus haute intensité quand il épaissit les références aux gestes et réactions indiennes aux traiteurs. Dans des explications limpides et souvent synthétiques, le chercheur montre sa longue familiarité avec la vision autochtone (aussi diverse soit-elle au niveau de chaque société). Dans le même mouvement, comme le souligne finement Carmen Bernand dans sa recension, Havard intègre dans sa grande histoire des circulations pelletières non seulement «les aspects relevant du développement commercial du trafic des peaux », ainsi que " des questions anthropologiques qui nous éclairent sur les valeurs et les comportements culturels des Amérindiens ", mais elle se réjouit, et nous avec elle, de ce que ce travail porte avec la même force sur «l'univers culturel des trappeurs français ». Elle regrette une certaine décontextualisation des Européens dans les études «coloniales" (c'est elle qui utilise des guillemets), " comme si ceux-là ne relevaient pas aussi des coutumes familiales, sociales, régionales et religieuses de leur époque, qui sont nécessairement affectées par les mutations historiques » (Bernand \$3). Contre l'interprétation souvent erronée des Euro-Américains qui y sont confrontés, les pratiques indiennes sont replacées dans leur contexte et montrent la grande distance, mais aussi la surprenante proximité, selon les moments et les lieux, entre les mondes européens et indiens. La sociabilité est insérée dans une vision religieuse amérindienne qui attribue à chaque Européen un caractère mystérieux, souvent puissant et surnaturel qu'il faut essayer de s'attribuer. Ainsi, on touche beaucoup ces hommes, leurs cheveux, leur visage, leurs mains et pieds que l'on masse, leur corps que l'on enduit d'onguents, ou plus prosaïquement de graisse. Ou bien on pleure sur eux dans « un acte de communion avec une altérité d'ordre surnaturel » (Havard 145). Encore, on les élève au moyen d'une peau par exemple, pour les décoller du sol et les transporter dans le village. Toutes ces pratiques ont un but : acquérir la puissance de l'étranger en se frottant, littéralement, à lui.

9 C'est cet objectif qui est le fondement de ce qui a été vu comme la liberté sexuelle des femmes amérindiennes. La facilité avec laquelle les Blancs avaient accès à ces femmes, même mariées, ou bien à des jeunes filles, qui étaient offertes par leurs époux, leurs pères ou leurs frères, aux visiteurs de passage. Les femmes étant liées au surnaturel et 
aux puissances naturelles par leur pouvoir d'enfantement étaient ainsi des médiatrices culturelles. Elles étaient loin d'être les simples victimes de ce système et remplissaient un rôle social assumé qui n'empêchait d'ailleurs pas les sentiments personnels de s'exprimer. Leur fonction de liant social est à maintes reprises soulignée dans le texte ${ }^{3}$. Havard montre qu'un système matrilinéaire aussi répandu dans les Grandes Plaines, il était difficile pour les traiteurs d'intégrer les groupes amérindiens sans obtenir un lien de parenté lié à une mère, une sœur ou une épouse. Père, fils, frère, mari, peu importait le titre, il fallait un lien de parenté pour être reconnu ou toléré. Bien entendu, cette position ouvrait également des devoirs dans la société indienne, en premier lieu l'obligation de générosité, sur laquelle était fondé le pouvoir des puissants. Nécessité très ironique et potentiellement ruineuse quand cela concerne des marchands avides de profit et d'enrichissement dans le monde capitaliste euro-américain! Mais, comme l'écrit Havard, la société amérindienne est "un monde où la sociabilité prime sur l'économie » (496), une conception très étrangère à la vision euro-américaine depuis quelques siècles déjà.

\section{La forme}

L'intérêt pour les récits de vie qui structurent cet ouvrage vise, malgré les trous, les bribes et les vides, non pas à une "restitution globale de la réalité", comme l'avançaient un peu audacieusement les ethnologues Jean Poirier et Simone ClapierVallladon en 1980, mais au moins « le roman vrai » (15) de Paul Veyne auquel se réfère Havard. Que l'histoire soit un récit avec l'homme pour acteur, selon la définition que Veyne donne en 1971, montre, pour reprendre l'expression de Mathieu Devinat, qu'elle " est à la fois art et activité savante »(\$5), une narration qui implique des faits, de la contextualisation, un recours aux autobiographies comme aux compte rendus de ceux qui ont côtoyé les personnages. "[C]omme il existe un décalage entre les faits et ce qu'en disent les documents, le roman de l'historien est une reconstitution du réel qui implique un recours à son imagination ", continue Devinat (\$8). L'ethnobiographie défendue par Poirier et Clapier-Valladon est une bonne description des pratiques d'historien auxquelles Havard a recours dans son travail : recherche sur « les modèles culturels du groupe »; " regard critique » sur la relation entre l'individu et le milieu ; enfin, relativisation de l'information en la recoupant avec "des enquêtes parallèles » (352). C'est à peu près exactement ce que décrit Havard quand il mentionne la «mise en récit des traces de singularité " «appuyée à un travail de critique et de contextualisation des sources » (16). L'auteur propose soixante-quatorze pages de notes souvent aussi passionnantes que le texte (513-586) et une bibliographie de trente-neuf pages qui comprend des sources primaires telles que lettres, relations, actes judiciaires, contrats de mariage ou d'engagement, inventaires, recensements, objets de la culture matérielle (16) et un nombre presque incalculable de sources secondaires de toutes les époques et de tous horizons (587-625). L'ouvrage contient des illustrations très nombreuses, dont de nombreux clichés de l'auteur qui aident à personnaliser ce travail au reste extraordinairement professionnel, comme lorsqu'il précise que «nous nous appuyons sur l'excellente biographie de Tykal tout en prenant soin de revisiter chacune des sources qu'il a identifiées et d'en mobiliser de nouvelles » (note 2, 570). On y trouve également un glossaire des peuples autochtones. L'appareil de notes souffre cependant 
d'un manque de repères du chapitre correspondant en fin d'ouvrage qui rend la consultation des notes très fastidieuse.

11 La mise en récit de cet énorme corpus est soutenue par une imagination d'écriture très puissante qui vient renforcer la force d'évocation de l'ensemble et de chaque partie. Ceci s'illustre brillamment à certains débuts de chapitre, tous très savoureux, qui sont souvent des reconstitutions d'une scène qui «aurait » pu se passer, ou qui s'inspirent d'un document iconographique que l'auteur contextualise et illustre par cette scénette fictive. Le roman vrai est pleinement assumé dans ces pages, le réel des situations prenant sa revanche sur la réalité absente de l'instant.

\section{Structuration de l'ouvrage}

Les vies de neuf personnages structurent l'ouvrage, dans un mouvement chronologique qui va de la fin du $\mathrm{Xv}^{\mathrm{e}}$ siècle à la fin du $\mathrm{XIX}^{\mathrm{e}}$. Trois d'entre eux ont rédigé des textes autobiographiques tandis que les autres, analphabètes, apparaissent ponctuellement dans les écrits et les descriptions de relations de voyage, ou dans les papiers officiels qu'a glanés Havard dans de nombreux fonds et manuscrits sur deux continents. L'auteur plonge dans l'existence de ces hommes au plus profond de ce qu'il est possible de faire suivant les sources. Les premières pages de chaque chapitre proposent systématiquement une réflexion méthodologique sur les sources et l'utilisation qu'il en fait, en plus du statut de ces sources, officielles, littéraires, ou artistiques.

Le livre est une somme qui devrait enthousiasmer toutes sortes de lectrices et de lecteurs et, on l'espère, de nombreux universitaires à l'avenir.

\section{Les chapitres}

14 Le premier chapitre commence avec la figure la plus fantômatique de toutes, celle de Pierre Gambie. L'action se passe en 1564. Le jeune homme a été laissé aux soins des Timucua (Floride actuelle) dans le but d'apprendre leur langue et de faciliter les échanges futurs avec les marchands européens. Gambie est un "truchement ", un «traducteur des mondes» (24). Son portrait apparaît dans un dessin de Jacques le Moyne Morgues, repris dans une gravure datée de 1591 de Theodore de Bry, le célèbre dessinateur, graveur et éditeur liégeois. Sur la gravure, il est sur le point d'être assassiné par un guerrier. Havard en reconstitue le récit, grâce notamment aux notes de Morgues, rescapé des massacres perpétrés par les Timucua et les Espagnols. Havard conclut que Gambie, dont nous savons si peu, est représentatif d'une caste de jeunes garçons qui a « complètement échappé... à la sagacité des chroniqueurs » (28).

Le deuxième chapitre concerne un autre truchement, Etienne Brûlé, "enfanté par l'aventure coloniale française" (35) et rescapé grâce aux écrits de Samuel de Champlain, d'un moine Franciscain, Gabriel $T$. Sagard, et du père Jésuite Jean de Brébeuf. Un tel placement d'enfant n'est pas étranger aux pratiques économiques européennes, remarque Havard (38). Brûlé est donc placé en 1610 par Champlain chez les Hurons et devient le "personnage-clé » de leur association avec les Français (43). Cette vignette insiste sur les évolutions des représentations sociales, notamment «la montée de la conscience civilisatrice et de l'ordre dévôt » qui jette progressivement sur les traiteurs un œil de plus en plus réprobateur, «ensauvagés » qu'ils sont par leur 
proximité avec les Amérindiens. L'opinion de Champlain est emblématique, mais là encore, les stratifications sociales tendent à voyager avec les immigrants, lorsque Havard écrit que « [p]our Champlain, la sauvagerie n'est qu'une version américaine et exotique de la culture populaire des campagnes les plus reculées » (64).

Héros du troisième chapitre, Pierre-Esprit Radisson (le " préféré » de Carmen Bernand, \$5, et qui suscite aussi l'admiration de Havard pour le corpus de six textes qui " possède[nt] une épaisseur narrative réjouissante », 86) est sans doute le personnage le plus truculent du recueil, un «formidable beau parleur » dont les aventures sont les plus frappantes, récit puisé aux sources de l'oralité comme la littérature de la gueuserie et le roman picaresque du $\mathrm{XVII}^{\mathrm{e}}$ siècle (87). Radisson est intégré dans un groupe d'Iroquois, la nation la plus crainte de la région. On peut dire que l'un des intérêts du personnage est «l'instabilité de son sentiment d'appartenance » (108) qui le conduit à trahir ses hôtes plusieurs fois, à subir la torture avant d'en être soigné. Mais Radisson est aussi une figure de la débrouillardise, de la ruse, de l'adaptation aux circonstances ( ( fais selon la mode »,111). Il incarne ainsi la figure autochtone du trickster, le filou, la figure de la transgression et de l'intégration (128). Il prend l'habitude de jeter de la poudre dans le feu pour que l'explosion sidère ses hôtes et étende son prestige (146). On trouve également dans ce texte une description glaçante de la famine à l'hiver 1660 et une évocation de ce qui fut sans doute la première rencontre européenne avec les Dakotas, les fameux Sioux dont les clichés représentent encore aujourd'hui dans l'imaginaire européen ce qu'est un Indien d'Amérique du Nord (143). Havard redéfinit des termes galvaudés tels que "Manitou ", ici une " personne autre qu'humaine dotée de pouvoirs spéciaux» (135-136) ou bien nous informe du mot dakota pour bison, tatanga. Ce chapitre est d'une richesse d'évocation, d'un foisonnement d'information et de réflexions anthropologiques tels qu'on aimerait y consacrer toute la recension. Le texte de Radisson est un "roman d'apprentissage et d'initiation" (90) dans lequel "l'instinct de survie est la clé » (154) du récit. Il fonctionne, écrit Havard, comme les autobiographies anglaises qui "justifient l'ascension sociale de leur auteur», un «traité de mobilité sociale » (138). La dernière vignette montre un Radisson de 74 ans, «mué en gentleman londonien », déambulant «avec sa canne et sa perruque dans les rues de West End » après avoir été « considéré à Versailles comme un traitre à la cause française » (160). Plus qu'un coureur de bois, Havard le voit comme un « entrepreneur » qui grâce à son expertise et en particulier sa connaissance des langues, a su naviguer dans un "contexte d'altérité » (161) et proposer à ses lecteurs une évocation de "l'Amérique des coureurs de bois comme aucun autre récit de cette époque » (162).

17 Le quatrième chapitre concerne "une de ces personnes d'expérience", "à la fois homme de terrain et homme de plume", dont les relations seront intégrées dans les textes de Claude-Charles le Roy de la Potherie et du jésuite Joseph-François Lafitau (170-171). Nicolas Perrot a été « accueilli chez les Pouteouatami (connus en anglais sous le nom de Potawatomi), à Michilimakinak, à l'ouest des Grands Lacs, au début du XVIII ${ }^{\mathrm{e}}$ siècle. Perrot est une sorte d'anti Brûlé et Radisson, Havard le décrit comme n'existant pas "contre le pouvoir - le pouvoir colonial - mais pour, par, ou avec» (166). Sa connaissance des langues et des mœurs pouteouatami le rendent longtemps précieux pour la couronne et apprécié de ses hôtes. Mais le chapitre est particulièrement intéressant concernant une double focalisation sur l'importance du tabac dans la sociabilité masculine, que les Indiens utilisent "lorsqu'ils veulent entrer en contact avec les esprits-animaux", et le culte du soleil chez les Pouteouatami que Havard 
rapproche de celui rendu au roi du même nom, à la même époque, en France. Mais l'historien fait un travail encore plus intéressant, quand il va chercher dans l'environnement dans lequel Perrot a grandi des représentations solaires qui auraient pu influencer l'intérêt que le voyageur avait à relater le culte de l'astre chez les Pouteouatami. Il mentionne par exemple une représentation solaire «sur le plafond à caissons de la salle des devises du collège [jésuite] des Godrans » (179) à Dijon dans lequel Perrot a fait ses études. Il insère également une photo d'un ostensoir d'argent appartenant à Perrot et retrouvé dans les ruines d'une ancienne mission dans la baie des Puants, qui représente l'astre solaire (181). Cela permet à Havard de montrer des concomitances entre les deux mondes et de s'éloigner (c'est son terme) « du registre interprétatif de l'acculturation, qui a longtemps dominé le champ des études amérindiennes ». Il donne pour exemple le «rituel mimétique " durant lequel les Indiens font visiter le village à Perrot en imitant la marche cadencée des soldats français, «de file deux à deux, le fusil sur les épaules" (186). Il précise un trait fondamental dans la relation autochtone aux étrangers " où l'imitation agit comme arme rituelle d'une pensée classificatoire hostile à la nouveauté et aux différences. Elle exprime, en définitive, la propension des Indiens à dissoudre et à absorber les étrangers - à les adopter d'une façon ou d'une autre " (187). Le but ultime de ces pratiques, conclut l'ethno-historien, est de «maintenir intactes leurs propres façons» (187). Havard s'intéresse également à "l'hospitalité légendaire des Indiens » qui a été tant vantée par les Européens, car pour lui, cette vertu « constitue une valeur fondatrice de la civilisation occidentale » (222), qu'elle soit issue de la noblesse ou du christianisme. À cette époque cependant, des jésuites comme Lafitau voient cette tradition "d'une économie traditionnelle des faveurs " (223) en Europe en train de s'effondrer sous l'influence du capitalisme naissant (222-223). L'honnête homme Perrot s'insurge des pratiques nouvelles qui font disparaître l'hospitalité et le don, accusant les coureurs de donner mauvaise réputation aux Français et se plaçant lui-même à un niveau bien supérieur (223). Ses dernières années le conduisent à gémir de ses déboires financiers, «à la croisée de deux cultures du dénuement »: celle des serviteurs désintéressés du roi et celle des Indiens qui « utilisent le langage de la pitié pour obtenir le soutien des esprits » (233).

Le cinquième chapitre s'ouvre sur un groupe d'adolescent-e-s qui découvrent en 1913 une " plaque de souveraineté » sur les bords du Missouri, dans le Dakota du Sud. Cette «tablette de plomb» (238) mentionne "L'an 26 du règne de Louis XV » et est signée «Pierre Gaultier de Lavendrie » (239). Havard produit deux photos de l'objet (239-240) suivant l'intérêt qu'il montre pour la culture matérielle de ses personnages. La plaque est conservée au Musée historique de Pierre, dans le Dakota du Sud. Au verso, on trouve quatre noms, celui de deux fils de la Vérandrye et ceux de deux hommes que Havard qualifie de "naufragés de la mémoire» (239), La Londelette et Amiotte. Les La Vérandrye ont entrepris un des premiers voyages européens dans les Grandes Plaines et demeurent oubliés jusqu'à ce qu'un archiviste français exhume en 1842 « des lettres et des résumés qui témoignent de l'épopée continentale des La Vérendrye » (241). Pierre Margry, c'est son nom, participe à ce que ces "découvreurs" des Rocheuses soixante-dix ans avant les premiers Mountain Men étatsuniens deviennent des « héros de la colonisation française » (241). Pourquoi les La Vérendrye se sont-ils aventurés si loin si tôt? Pour tenter d'y découvrir un passage vers l'ouest, le fameux chenal qui lierait l'Amérique à l'Asie. Jefferson enverrait Lewis et Clark à la poursuite de cette chimère euro-américaine en 1804 pour découvrir que ce passage mythique n'existe 
pas $^{4}$. Contrairement aux récits de Radisson, les La Vérendrye bénéficient d'une hospitalité chaleureuse où qu'ils se présentent, particulièrement chez les Arikaras (les gens de l'arc), dont ils apprécient «les belles manières" de leur chef (265). Il se pourrait comme le suggère Havard que les Blancs soient dans cette période de premier contact perçus comme "des personnes autres qu'humaines ", ce qui leur aurait procuré " une relative immunité » (263). Ils rencontrent également les gens des Chevaux, dont Havard pense qu'il pourrait s'agir des Cheyennes, « ou šahiyena » (256), premier peuple des Plaines du Nord "à disposer de chevaux» ou bien "un groupe de troqueurs d'équidés» (257). La thématique saisissante de ce chapitre est sans doute la démonstration d'une présence démographique « dans l'histoire pré-épidémique de la région» (266). Havard relate un déplacement massif d'Arikara (auxquels se joignent d'autres tribus) à l'occasion d'une campagne de guerre « contre les Serpents " (connus en anglais sous le nom de Shoshone, 511). La Vérendrye compte « 2000 combattants » et avec les familles, Havard avance le chiffre de dix mille personnes marchant dans les Plaines, donnant l'image surprenante à nos yeux habitués aux représentations « de la faiblesse démographique » $\mathrm{du} \mathrm{XIX}^{\mathrm{e}}$ siècle d'une «horde nomade qui se déploie dans la prairie giboyeuse sur plusieurs kilomètres » (266). Le terme « horde » est ici surprenant si l'on pense notamment aux enfants qui la composent. Un autre thème important de la vie régionale est l'arrivée du cheval dans les Plaines du Nord. Ce nouveau moyen de locomotion et de transport des marchandises est une révolution technique que Havard appelle « un adjuvant de la guerre » puisque «sa capture rentre dans la hiérarchie des honneurs guerriers » et rend les conflits plus meurtriers (264). Dans ce contexte de tensions extrêmes, les appels au calme semblent vains et le discours des agents de la «Pax Gallica » que sont les La Verendrye est tout bonnement jugé «irréel » (267). La plaquette de plomb qui clamait la grandeur de Louis $\mathrm{XV}$ et son emprise sur ces contrées a fini enterrée pour n'être redécouverte que cent soixante-dix ans plus tard.

Dans le sixième chapitre, Jean-Baptiste Truteau travaille pour une compagnie pelletière qui a obtenu l'exclusivité du commerce dans le haut Missouri, avec pour objectif la plaque tournante du commerce que sont les villages mandanes du Dakota du Nord actuel (280). Il sait écrire et compose des textes à destination de ses employeurs et du Lieutenant-Gouverneur de la Haute-Louisiane encore sous commandement espagnol. L'action débute en 1794 (281). À l'instar de Perrot, Truteau est « expert et victime du pays indien » (281) et se plaint régulièrement de ne pas être reconnu à sa juste valeur. Havard voit donc dans son écriture un "exutoire» à ses frustrations (283). Ses multiples voyages font de lui un voyageur représentatif de sa confrérie et de leur indifférence aux "nouvelles frontières officielles". Truteau s'installe donc comme "traiteur", voyageur professionnel, à St Louis, ville pour laquelle le commerce des peaux est au «fondement de son économie » (292). Il parle le sioux yankton et le pani pour avoir séjourné avec ces tribus dans les années 1770 et 1780 (293). S'il maitrise quelques langues, Truteau a pourtant un gros défaut: il n'apprécie pas la culture du don et essaie souvent de se défiler. Ainsi, sa pirogue chargée " viole l'une des règles les plus élémentaires de la sociabilité " quand la masculinité "est associée à l'immatérialité chez les Sioux » (303). Un épisode très drôle et effrayant est celui où des " hommes et femmes de tous âges, enfants compris... montent dans la pirogue et se servent sans ménagement » (303). Le traiteur reçoit des peaux en échange, car ce qu'il nomme "pillage » est à la fois la libéralité normale de celui qui a (le Big Man) envers ceux qui manquent et une transaction, mais comme le précise Havard, «les termes en sont imposés par les Indiens» (304). D'ailleurs, une des figures indiennes les plus 
impressionnantes est Toangarest, L'Oiseau Noir, dont Truteau affirme qu'il est « le plus grand coquin de toutes les nations qui habitent le Missouri» (310). Ce chef «charismatique » est toujours sur son cheval qui, nous indique Havard, "plutôt qu'un moyen de locomotion commode, constitue un vecteur d'élévation, comparable à une litière rituelle " (311). L'auteur suggère que l'adoption du cheval dans les Grandes Plaines pourrait être économique et religieuse (311). La rancœur de Truteau ne permet sans doute pas d'évaluer correctement les qualités de chef de Toangarest. Les «tribulations» de Truteau (c'est le terme du titre du chapitre) sont une suite incessante de situations limites dans lesquelles il risque à chaque instant la mort, soit aux mains des Sioux, soit par manque de nourriture. Un des thèmes récurrents qui est bien illustré dans cette partie est l'alternance de périodes maigres et de périodes fastes, « de la disette au festin le plus gargantuesque », écrit Havard (307). Le livre est rempli de descriptions de banquets ou d'invitations à manger dans les tipis (pas moins de vingt références dans l'index, 635) dans lesquelles on mange des heures durant d'énormes quantités de viande. L'entrée «Faim/famine/disette » comporte douze références, montrant que l'accès à la nourriture était incertain, particulièrement à la sortie de l'hiver. À ces calamités habituelles s'ajoutèrent des épisodes épidémiques sévères qui réduisirent drastiquement les populations, comme l'épidémie de variole de 1780-1781 qui provoqua « une chute démographique de $68 \%$ » chez les Arikaras, Gros Ventres et Mandanes (note 83, 554). Les regroupements qu'entraînent ces cataclysmes, mais aussi des jeux d'alliance temporaire, rendent la compréhension de l'organisation humaine des Grandes Plaines difficile et passionnante, avec «un enchevêtrement de communautés indépendantes en flux constant et aux alliances aussi variées que changeantes" (326). Finalement, Truteau n'étant ni un guerrier ni un homme généreux, il ne s'inséra pas facilement dans les tribus. Il ne remplissait pas les conditions qui établissaient la réputation d'un homme : «la bravoure, la générosité, l'endurance et l'intégrité » (note 112, 556). Il passera la dernière partie de sa vie comme instituteur et précepteur « autoritaire » (334).

Le septième chapitre rend compte de la vie d'un homme qui doit son "grain d'historicité »(entendez sa place dans l'histoire) surtout à sa participation à l'expédition de Lewis et Clark, dont « l'aura mémorielle » imprime une « extraordinaire marque... sur tous les individus concernés de près ou de loin par l'expédition » (340). Toussaint Charbonneau prend corps dans les sources, nous dit Havard, grâce aux "observateurs-voyageurs qui ont croisé sa route ", car l'homme ne savait pas écrire (340). Il est aussi connu pour avoir été le mari de Sakakawia (Femme-Oiseau), l'unique femme et mère de famille de l'expédition mythique (341). Charbonneau n'a pas très bonne presse et Havard résume sa piètre réputation ainsi: "c'est donc presque toujours sur l'autel de l'infâmie que ce personnage s'est vu reconnaître une place dans la mémoire historique et folklorique étatsunienne » (341). Est-ce pour cela que l'on sent chez Havard le désir de réhabiliter au moins un peu celui dont il dénonce l'utilisation à « valoriser... l'impeccable moralité et le courage sans failles des Américains »? (341).

En tout cas, ce type d'effacement fait écho à celui contre lequel il écrit son ouvrage et on sent qu'il a tenté dans ce chapitre une réhabilitation au moins partielle d'un « individu à l'endurance étonnante et qui semble avoir fait le choix de vivre parmi les Indiens » (342), c'est-à-dire les Gros Ventres. Il ajoute qu'on peut voir dans «les appréciations négatives qui visent le Canadien une marque coutumière du mépris des officiels américains ou écossais à l'égard des rudes interprètes francophones » (386). Toutefois, l'honnêteté de l'auteur apparaît lorsqu'il mentionne un épisode de violence 
sexuelle, ou ce qui s'y apparente. Charbonneau semble avoir été un homme très attiré par les femmes amérindiennes. D'ailleurs, l'un de ses cinq surnoms gros ventres est «vagin gargouilleur » et Havard indique qu'il ne semble jamais avoir connu le célibat (343-344; 379 ; 388). Au tournant du XIx ${ }^{\mathrm{e}}$ siècle, il vivait avec deux femmes (dont Sakakawia), un arrangement réservé "aux hommes les plus prestigieux", ce que Havard interprète comme le signe d'un certain «statut social » peut-être autant que d'un «confort personnel» (346). Cependant, les femmes amérindiennes n'étaient pas de pauvres victimes sans défense, leur « indépendance » et leur « volonté de regagner leurs familles » suscitait «bien des frustrations chez leurs compagnons blancs », nous informe l'auteur ${ }^{5}$ (395).

Il y aurait beaucoup à dire de l'expédition, mais je retiendrai ici la chaîne de traduction, parfois impliquant trois ou quatre langues, pour parvenir à communiquer entre les capitaines anglophones et Cameahwait, le chef serpent, frère de Sakakawia (anglais, français, gros-ventre, serpent, 357). Charbonneau est décrit comme un «excellent cuisinier ", ce qui lui confère un statut sans doute particulier parmi ses compagnons. Havard nous donne la recette du boudin de bison, qualifié de " plus grand délice de la forêt » par Meriwether Lewis. Sakakawia, elle, excelle à la « collecte de végétaux » qui complètent l'alimentation carnée du groupe (353). L'intérêt particulier du personnage de Charbonneau est son attachement viscéral à la liberté : pour lui, « la liberté n'est pas un principe intellectuel », écrit Havard, "c'est un principe vécu » (365). Il est en effet l'un des rares, et le seul de cette sélection, à avoir choisi de vivre chez les Gros Ventres de façon permanente. Sakakawia meurt en 1812 et William Clark adopte les deux enfants Charbonneau définitivement. Toussaint poursuit sa carrière de médiateur en devenant interprète pour l'armée et travaille épisodiquement pour des visiteurs fortunés comme le Prince Maximilian. Celui-ci semble avoir beaucoup apprécié Charbonneau et donne de très précises indications à son sujet, « tireur d'élite ", « fort ", « robuste » (386).

Enfin, ce chapitre est intéressant par une des analyses anthropologiques dont Havard a le secret, ici à propos du bison. Nous apprenons que cet animal se définit "par une présence associée à des agissements particuliers ». Ainsi, les vieillards qui imitent leurs agissements en dansant acquièrent l'« agir-bison", des "capacités performatives qui visent à la multiplication des troupeaux » (388). Comme ce fut le cas cinquante-sept ans plus tôt, la variole décime à nouveau les Mandanes et les Arikaras en 1837 et 1838, tuant 90\% de la population dans le village de Mih-Tutta-Hang-Kush qui passe de mille six cents à trente résidents (398). Havard conclut : « [s]ous les yeux de Charbonneau, une civilisation flamboyante s'évanouit» (398). Disons pour tempérer cette vision du «Vanishing Indian » qu'une phase majeure de l'histoire dakota est en train de prendre fin.

Le huitième chapitre est consacré à Étienne Provost, un «Falstaff francophone » qui " construit sa propre légende» $(431,435)$. Provost est un chasseur familier des Montagnes Rocheuses à partir des années 1830. Ce chapitre est singulier par la place qu'y tient un peintre, Alfred Jacob Miller, dont les œuvres «sont les seules représentations visuelles des trappeurs qui soient contemporaines des 'rendezvous' ", ces grandes foires qui rassemblaient des centaines, voire des milliers de personnes, coureurs et Amérindien-ne-s (404). Ce chapitre est donc illustré très efficacement par les œuvres de Miller et d'autres témoins de cette vie des Grandes Plaines, dont un beau dessin d'un officier britannique (448). Miller a peint Provost et son ventre généreux. 
C'est la description enlevée de son tableau qui ouvre le chapitre. Le tableau de 1837 est en double page dans le livret au milieu de l'ouvrage. L'intérêt ici est la démocratisation en Amérique de deux pratiques qui ont toujours été farouchement réservées à l'aristocratie européenne : la chasse et le cheval (435).

Ce chapitre évoque de multiples transformations par rapport aux précédents. L'évolution de la ville de St Louis vers 1850 perd son caractère francophone avec l'arrivée de nombreux Irlandais et Allemands (445). C'est aussi la fin des pelleteries, avec l'extinction du castor dans les Rocheuses vers 1840 (436) après sa disparition de la partie orientale du continent. Havard décrit précisément la technique de "pêche » du castor, notamment à l'aide du "castoreum » une sécrétion glandaire qui attire les animaux vers le piège. Perçus uniquement comme la source du feutre extraordinaire que l'on extrayait de leur fourrure, on a oublié que les castors sont d'extraordinaires " ingénieurs d'écosystème " (Wohl 1). Cependant, une scène extraordinaire est celle à laquelle assiste Provost et ses compagnons quand une harde de bisons défile devant la caravane durant trois jours et trois nuits sans interruption. La démesure des Plaines trouve une autre illustration dans la description d'un campement de Sioux Yankton de trois cents tipis. (436) La mobilité sera pour Provost « synonyme de notoriété » (409) et Havard est catégorique : «Provost n'a jamais cessé d'être un voyageur » (405).

Son aire d'activité est impressionnante, mais typique des grandes expéditions de voyageurs à l'Ouest du Mississippi : sont couverts les territoires actuels du Nebraska et du Colorado, mais aussi l'Arkansas, jusqu'à Santa Fé (ville espagnole, puis mexicaine, créée en 1607-1610). "Il est le premier trappeur euro-américain » à voir le Grand Lac Salé d'Utah (412). Alors, sommes-nous plongé-e-s dans la conquête de l'Ouest? Non, répond Havard, car cette activité frénétique, ce sillonnage incessant des pistes, ces échanges difficiles avec les autochtones ont lieu dans « des territoires qui demeurent sous la souveraineté des Indiens » (405). Provost est un entrepreneur et à cet égard, il ne se contente pas du salaire confortable qu'il touche de ses employeurs et de ses extras en tant que guide et expert de l'Ouest. "Il est désormais à la tête d'un saloon » qui sert de lieu de convivialité et de sociabilité aux voyageurs durant l'hiver et lui sert également de base arrière pour l'organisation de "ses expéditions dans le pays indien " (412). Un autre lieu de rencontre et de sociabilité est le rendez-vous, "un défouloir proprement masculin, où se mettent en scène les membres d'une confrérie saisonnière » (428). Le rendez-vous, précise Havard, est « un théâtre social structuré autour de l'idée de fête permanente et de dépense improductive " (429). L'accent est donc mis sur la question de la "dilapidation monétaire ", une sorte d'émancipation de ces hommes par rapport au système capitaliste auquel ils sont quand même inféodés, une pratique de libéralité hérité des Amérindiens (429). Provost y croise d'ailleurs JeanBaptiste Charbonneau, le fils de Sakakawia et Toussaint (428). Un autre personnage célèbre l'engage pour une expédition, John James Audubon. Cette page dénonçant des pratiques de chasse sans discernement est l'une des rares dans lesquelles on sent Gilles Havard scandalisé par ce qui «devient autant un safari américain qu'une campagne scientifique ». Audubon et ses hommes " font feu sur le moindre animal entrant dans leur champ de tir, du sport et de l'aventure » (458). On sent bien ici que ce genre de pratique est en train de faire glisser l'époque, déjà mortifère pour les castors et les bisons, vers l'anéantissement d'une certaine forme de biodiversité dans les Grandes Plaines. L'auteur se garde néanmoins de commenter sur cette question. 

Dakota du Nord, chez les Mandanes et les Gros Ventres. Il concerne un interprète de l'armée américaine, Pierre Beauchamp, âgé de cinquante-huit ans. La scène se passe en 1867 et Havard nous donne une liste des effets à présent très prononcés de l'invasion euro-américaine des Grandes Plaines. Il introduit dans son récit un autre français, officier breton de l'armée étatsunienne, vétéran de la guerre de sécession récente, Régis de Trobriand. Le premier dialogue de cette dernière partie est donc la rencontre de ces deux hommes dans cet «univers francophone, comme enchâssé dans le pays indien » (475). Havard poursuit sa brillante démonstration de la place du français dans ces contrées à présent dominées par la présence étatsunienne, jusque dans la seconde partie du XIX $x^{e}$ siècle. Beauchamp est à contre-courant de l'évolution générale de la société quand il déclare que «les villes, ça ne me va pas... je mourrai où j'aurai vécu, dans la prairie » (475). Un autre signe de la disparition d'un certain mode de vie hérité des valeurs et pratiques amérindiennes des Grandes Plaines. L'homme est marié à une Arikara nommée Femme-Tabac (Staawiškaánu) (481). Peu indianisé, il se « revendique d'appartenir à la communauté arikara » (482).

Les sources qui témoignent de la vie de ce voyageur sont issues des écrits de Trobriand et d'une entrevue de Beauchamp avec l'anthropologue Louis Henry Morgan qui l'interroge en 1862 à Fort Berthold sur la parenté indienne (481). Pierre Beauchamp a eu un fils avec Femme-Tabac, appelé Kaniítu, rocher, pierre, qui est interprète pour l'armée également. Havard en produit une photo où le jeune Pierre pose en 1874 avec un grand collier autour du cou et une besace, aux côtés du Chef arikara Son of the Star, durant une visite officielle à Washington (485). Havard termine ce très court chapitre en soulignant que "la souveraineté des autochtones ne sera bientôt plus qu'un souvenir dans les Plaines du Nord » (484). Là encore pourrait-on espérer un lien plus évident avec la situation d'aujourd'hui qui rend un peu de cette auto-détermination en partie retrouvée aux groupes Dakota actuels.

Il termine ce chant du cygne par un bond vers le temps présent. En 2007, il rencontre avec son ami anthropologue Douglas Park une arrière-petit fille de Pierre Beauchamp, à Fort Berthold, qui se souvient de son aïeul et «s'identifie comme une Indienne». Un autre descendant rencontré par l'auteur et son ami leur tient le même discours sur les origines allemande, irlandaise, française et arikara de la famille, mais il leur déclare : « je ne suis pas indien. Je regarde devant moi, pas derrière » (486). Havard trouve une multitude de patronymes français dans les registres de la réserve arikara. Il conclut en disant que «le passé franco-indien... ne subsiste que dans l'intimité des récits familiaux » (486).

\section{Remarques et conclusions}

Trois notions auront donc présidé à ce travail, conclut Havard, la mobilité, la masculinité et les rencontres interculturelles (487). Tous les personnages étudiés ici sont " atypiques " car pour la plupart des coureurs de bois, "la règle, c'est surtout d'échapper aux sources narratives ", écrit-il (492). Havard insiste sur la porosité des milieux en présence. Ces hommes sont capables de transcender les déterminismes et de naviguer dans les eaux tumultueuses des mondes euro-américains et indiens, usant de compétences transnationales, adoptant parfois les techniques amérindiennes qui consistaient à profiter des antagonismes impériaux pour défendre au mieux leurs

Transatlantica, 1 | 2021 
propres intérêts, à l'image de Radisson qui finit sa vie à Londres pour échapper aux autorités françaises. L'indétermination du monde des coureurs de bois a bien sûr tendance à disparaitre à mesure que les états euro-américains affermissent leur emprise sur les territoires et relèguent la souveraineté autochtone à peau de chagrin à la fin du xix ${ }^{e}$ siècle. Havard remarque à ce propos qu'« une relative cohabitation des cultures n'empêche pas en effet les contrecoups délétères de la rencontre » (499). Ces trappeurs et coureurs de bois sont quand même des agents impériaux engagés dans une vaste opération de destruction des bêtes à fourrures, quel que soit le degré d'indépendance qu'ils cherchent à acquérir par rapport aux autorités ou même à leurs liens de parenté avec les sociétés amérindiennes.

On pourra ajouter que ce travail s'inscrit dans la continuité des acquis de la nouvelle histoire de l'Ouest qui, à partir des années 1980, décentra le mouvement de colonisation par les Euro-Américains vers une diversité de groupes ethniques et culturels (sans nier l'importance de la migration européenne venue de l'atlantique), réaffirma la diversité des nations indiennes, la complexité et les interactions de leurs cultures, ce à quoi contribue largement Havard dans son livre. On pourra même dire qu'il enrichit grandement l'historiographie du monde métissé franco-indien. La nouvelle histoire fit aussi émerger le rôle des femmes, les questions de classes sociales au sein d'une société soi-disant égalitaire, révélant l'influence grandissante de grandes sociétés nationales et transnationales dans le développement économique de la région.

On peut peut-être aussi s'interroger sur un certain sens d'une fin d'époque après les années 1850 , validant implicitement la thèse de la disparition qui fait fi de la résistance et du retour amérindien sur les scènes politiques locale et nationale à partir des années $1970^{6}$. La question d'identifier la région connue par les non-autochtones comme «le pays d'en haut" sans en référer aux points de vue autochtones est une question récurrente qui fait couler beaucoup d'encre depuis longtemps (Monette-Deschênes, 2018). Plus généralement, on aurait pu s'attendre à davantage de recours à des références à l'épistémologie autochtone en plein développement ces dernières années (Hickey, 2020 ; Held, 2019). L'agentivité amérindienne est évidente dans le texte, mais comme le dit d'ailleurs Gilles Havard à propos des Indiens de Floride au $\mathrm{xvI}^{\mathrm{e}}$ siècle, ceux-ci ont longtemps eu " une existence quasi-fictive » (29) et le texte gagnerait sans doute à équilibrer davantage l'effort de matérialisation (de dé-fictionalisation ?) qui est produit envers les coureurs de bois, peut-être comme il le fait très succinctement à deux reprises avec des descendants arikara, en allant du côté de l'histoire orale amérindienne qui apparaît peu dans les sources proposées (je n'ai relevé qu'une référence à l'association des descendants de Nicolas Perrot (587) et les notables références aux anthropologues tels Raymond DeMallie ou Douglas Parks $(597,614)$. Je tempérerai cette remarque en soulignant la présence continue de traduction de noms, voire de mots ou de concepts amérindiens tout au long de l'ouvrage. Le portrait d'un chef comme L'Oiseau Noir nous fait entrevoir une approche des personnalités amérindiennes qu'on aurait sans doute aimé rencontrer davantage dans l'ouvrage.

Il n'en demeure pas moins que L'Amérique fantôme est un travail majeur qui poursuit l'œuvre entamée depuis maintenant près de trente ans par Gilles Havard et par les ethnohistorien-ne-s états-unien-ne-s de la Nouvelle Histoire de l'Ouest à partir des années 1980 ainsi que les travaux canadiens de chercheur-e-s comme Denis Delâge, Bruce Trigger ou Pierrette Désy. La contribution de ce travail à la compréhension des 
dynamiques internes et externes ainsi que des visions du monde, propres aux voyageurs et aux Amérindien-ne-s qui les ont côtoyés, est immense.

\section{BIBLIOGRAPHIE}

BERNAND, Carmen. « Gilles Havard, L'Amérique fantôme. Les aventuriers francophones du Nouveau Monde ». Nuevo Mundo Mundos Nuevos [En ligne], Comptes rendus et essais historiographiques, mis en ligne le 08 octobre 2019. journals.openedition.org/nuevomundo/ 77560. Page consultée le 12 février 2021

CATTON, Theodore. Rainy Lake House: Twilight of Empire on the Northern Frontier. Baltimore : Johns Hopkins University Press, 2017.

DEVINAT, Mathieu. « Réflexion sur l'apport de l'ouvrage. Comment on écrit l'histoire sur la formation à la recherche en droit ». Les Cahiers de droit, vol. 52, n 3-4, 2011, p. 659-670.

GITLIN, Jay. The Bourgeois Frontier: French Towns, French Traders, and American Expansion. The Lamar Series in Western History, Londres : Yale University Press, 2010.

GREER, Allan. « GILLES HAVARD, Histoire des coureurs de bois. Amérique du Nord 1600-1840, Paris, Les Indes savantes, 2016, 885 p., ISBN 978-2-84654-424-5 ». Revue d'histoire moderne \& contemporaine, vol. 66-2, n² 2, 2019, p. 188-190, https://www.cairn.info/revue-d-histoire-moderne-etcontemporaine-2019-2-page-188.htm. Page consultée le 13 février 2021.

HAVARD, Gilles. « Richard White, Le Middle Ground. Indiens, Empires et Républiques dans la région des Grands Lacs, 1650-1815 ». Revue d'histoire moderne \& contemporaine, vol. 57-1, n 1, 2010, p. 204-206.

HAVARD, Gilles. «Les Pays d'en haut, un espace en mal d'histoire? ». Francophonies d'Amérique, $\mathrm{n}^{\circ} 40-41,2015$, p. 19-54.

HELD, Mirjam B. E. « Decolonizing Research Paradigms in the Context of Settler Colonialism: An Unsettling, Mutual, and Collaborative Effort ». International Journal of Qualitative Methods, vol. 18, 23 January 2019.

HICKEY, Dana. « Indigenous Epistemologies, Worldviews and Theories of Power ». Turtle Island Journal of Indigenous Health, vol. 1 n 1, 2020, p. 14-25, https://doi.org/10.33137/tijih.v1i1.34021. Page consultée le 13 février 2021.

KNOWLTON-LE ROUX, Laura. « Sacajawea vs. Charbonneau ». Transatlantica [En ligne], 1 | 2006, mis en ligne le 25 mars 2006, URL : http://journals.openedition.org/transatlantica/300. Page consultée le 19 avril 2021.

MONETTE-DESCHÊNES, Audrey. " Décoloniser la terminologie autochtone : Une lettre ouverte ». Radio Canada, 17 avril 2018, https://ici.radio-canada.ca/espaces-autochtones/1095562/langueautochtone-nations-francais-decolonisation. Page consultée le 13 février 2021.

PERREAULT, Olivier. Enjeux postcoloniaux et nationaux dans les polémiques entre ethnohistoriens du Pays d'en Haut (1985-2016), Mémoire de Maîtrise ès arts en Histoire, Université de Montréal, 2020, https://papyrus.bib.umontreal.ca/xmlui/bitstream/handle/1866/23442/ 
Olivier_Perreault_2020_Memoire.pdf?sequence=2\&isAllowed=y. Page consultée le 13 février 2021.

POIRIER, Jean, et Simone CLAPIER-VALLADON. « Le Concept d'ethnobiographie et les récits de vie croisés ». Cahiers Internationaux de Sociologie, vol. 69, 1980, p. 351-358. www.jstor.org/stable/ 40689920. Page consultée le 13 février 2021.

PARKS, Douglas R. « The Importance of Language Study for the Writing of Plains Indian History ». New Directions in American Indian History. Dir. Colin G. Calloway. Norman : University of Oklahoma Press, 1988, p. 153-197.

SMITH, Andrea. « Not an Indian Tradition: The Sexual Colonization of Native Peoples ». Hypatia, vol. 18, n² 2, 2003, p. 70-85. JSTOR, www.jstor.org/stable/3811012. Page consultée le 19 avril 2021.

THWAITES, Reuben Gold. «Jefferson's Instructions to Lewis ». Original Journals of the Lewis and Clark Expedition, 1804-1806, vol. 7, New York : Dodd, Mead and Company, 1905, 247-52, Center for the Study of the pacific Northwest, https://www.washington.edu/uwired/outreach/cspn/ Website/Classroom\%20Materials/Reading\%20the\%20Region/Discovering\%20the\%20Region/ Texts/9.html. Page consultée le 12 février 2021.

WOHL, Ellen. « Legacy effects of loss of beavers in the continental United States ». Environ. Res. Lett. $\mathrm{n}^{\circ}$ 16, 2021, https://iopscience.iop.org/article/10.1088/1748-9326/abd34e/pdf. Page consultée le 12 février 2021.

WULF, Naomi. « Biographie et histoire dans la jeune République ». Transatlantica [En ligne], 1 | 2002, mis en ligne le 30 juin 2006, URL : http://journals.openedition.org/transatlantica/392. Page consultée le 14 février 2021.

\section{NOTES}

1. On trouve également dans cette collection Bronwen McShea, Apostles of Empire: The Jesuits and New France, 2019 ou encore Jonathan K. Gosnell, Franco-America in the Making: The Creole Nation Within, 2018.

2. Le titre de son dossier d'Habilitation à Diriger des Recherches en 2013 était «Amérindiens, Empires, coureurs de bois : éléments pour une ethnohistoire de l'Amérique du Nord (XVII ${ }^{\mathrm{e}}-\mathrm{XIX}{ }^{\mathrm{e}}$ s.) ".

3. On pourra cependant regretter l'absence de références plus explicites au travail d'un nombre grandissant de chercheuses concernant les rapports entre le colonialisme et les violences sexuelles. La fonction sociale des relations sexuelles, toute indéniable qu'elle soit dans certaines circonstances, ne peut occulter la récurrence des violences faites aux femmes depuis l'arrivée des conquistadores dans les Amériques, jusqu'au scandale des disparitions et meurtres de femmes autochtones à la période contemporaine. Voir par exemple Andrea Smith.

4. "The interesting points of the portage between the heads of the Missouri \& the water offering the best communication with the Pacific Ocean should also be fixed by observation, \& the course of that water to the ocean in the same manner as that of the Missouri" (Thwaites 247-252).

5. Comme souligné dans une note précédente, une contextualisation plus explicite de la violence sexuelle imposée aux femmes amérindiennes aurait été bienvenue. Un article de 2006 publié dans ces colonnes et rédigé par Laura Knowlton-Le Roux exprime cependant un point de vue assez similaire à celui de Havard sur l'aspect répulsif du coureur de bois francophone qu'est Charbonneau dans la construction du mythe états-unien: "I will argue that an aspect of the creation of the Sacajawea myth which has been overlooked, the unflattering portrayal of her husband Toussaint Charbonneau, actually played a central role in its construction. Charbonneau 
the "squaw man" became just as much a myth as Sacajawea in the expedition novels; he too was endowed with a disproportionate share of importance, but because he was used to represent the French threat to the Americans. The vilification of Charbonneau is common to expedition fiction, with only one exception" (\$3).

6. On se reportera à une discussion fouillée de la position de Gilles Havard par rapport aux débats entre ce que Olivier Perreault appelle les collectifs franco-québécois, le collectif ethnohistorien anglo-américain et le collectif autochtone, implicitement la question du point de vue et de l'épistémologie autochtones dans les études amérindiennes.

\section{AUTEURS}

\section{JEAN-MARC SERME}

Université de Bretagne Occidentale, Laboratoire HCTI 